\title{
Lesiones de mancha blanca en pacientes con tratamiento de ortodoncia. Revisión de la Literatura
}

\section{White spot lesions in patients with orthodontic treatment. Literature review.}

\author{
Marco Antonio Sanchez-Tito ${ }^{\text {1,a;2,b }}$, Lidia Yileng Tay Chu Jon ${ }^{2, c}$
}

\section{RESUMEN}

Las lesiones de mancha blanca son efectos adversos frecuentes en los pacientes con tratamiento de ortodoncia. Son producto del ataque de ácidos orgánicos capaces de desorganizar la estructura del esmalte y su formación está asociados a la presencia de factores de riesgo como el tiempo prolongado de tratamiento ortodóntico, la mala higiene bucal y edades tempranas de inicio de tratamiento. En la literatura existen diversos métodos de diagnóstico para este tipo de lesiones, así como diversas propuestas para su prevención y/o tratamiento. El objetivo de esta revisión de literatura es presentar la evidencia científica relacionada a la formación de las lesiones de mancha blanca, su prevalencia, factores de riesgo asociados a su formación, así como describir los métodos de diagnóstico más frecuentes y los mecanismos de prevención y de tratamiento.

PALABRAS CLAVE: Ortodoncia, mancha blanca, caries dental.

\section{SUMMARY}

White spot lesions are common side effects in patients with orthodontic treatment. They are the product of the attack of organic acids capable of disorganizing the structure of the enamel and its formation is associated with the presence of risk factors such as prolonged orthodontic treatment time, poor oral hygiene and early treatment initiation ages. In the literature there are various diagnostic methods for this type of lesions, as well as various proposals for prevention and/or treatment. The aim of this literature review is to present the scientific evidence related to the formation of white spot lesions, their prevalence, risk factors associated with their formation, and to describe the most frequent diagnostic methods and mechanisms of prevention and treatment.

KEYWORDS: Orthodontics, white spot, dental caries.

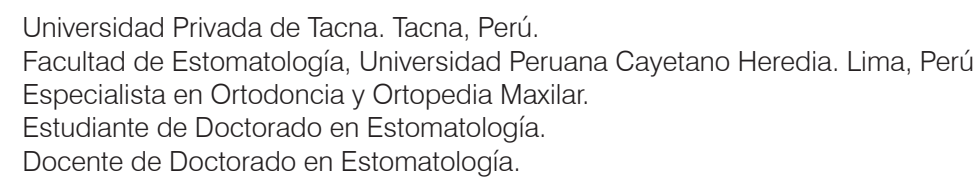




\section{INTRODUCCIÓN}

Las lesiones de mancha blanca (LMB) son uno de los principales efectos adversos del tratamiento de ortodoncia con aparatos fijos $(1,2)$. Su presencia muestra un desbalance del proceso normal entre desmineralización y remineralización de la superficie del esmalte. Estas lesiones pueden ser definidas como una porosidad en la sub-superficie del esmalte debido a la desmineralización por ácidos orgánicos (3).

Los pacientes en tratamiento de ortodoncia pueden presentar un alto riesgo de desarrollar LMB, existiendo factores de riesgo para su formación, como el mal cumplimiento del tratamiento, higiene bucal deficiente, tiempo prolongado de tratamiento y la edad de los pacientes. Estas características hacen que las LMB sean frecuentes en estos pacientes, alcanzando prevalencias de hasta el $84 \%$ según diversos autores; la forma en que se evalúa las lesiones de mancha pueden afectar estos valores, siendo que existen estrategias clínicas, el uso de fotografías o dispositivos y técnicas que permiten identificar la presencia de LMB en estadios tempranos.

En cuanto al manejo de las LMB existen diversas propuestas como la aplicación tópica de flúor, fosfato de calcio amorfo, aplicación de sellantes e incorporación de agentes antibacterianos a los cementos ortodónticos con la intención de evitar o prevenir su formación.

El objetivo del presente trabajo es describir la evidencia científica relacionada a los procesos de formación de las lesiones de mancha blanca, particularmente en pacientes ortodónticos, su prevalencia y los factores de riesgo asociados, además de describir los métodos de diagnóstico más frecuentes y los mecanismos de prevención y de tratamiento de este tipo de lesiones.

\section{Estrategia de búsqueda}

La revisión de literatura se realizó en las bases de datos: Pubmed, SciELO y Scopus. Las palabras claves empleadas fueron: white spot lesions, orthodontic treatment, prevention y sus combinaciones. Se incluyeron estudios observacionales, estudios experimentales in vitro, estudios tipo ensayos clínicos, artículos de revisión y revisiones sistemáticas. No se limitó la búsqueda por fecha de publicación. Adicionalmente, se incluyó la información contenida en un libro.

\section{Formación de las lesiones de mancha blanca}

Las bacterias contenidas en el biofilm producen ácidos no disociados producto de la exposición a

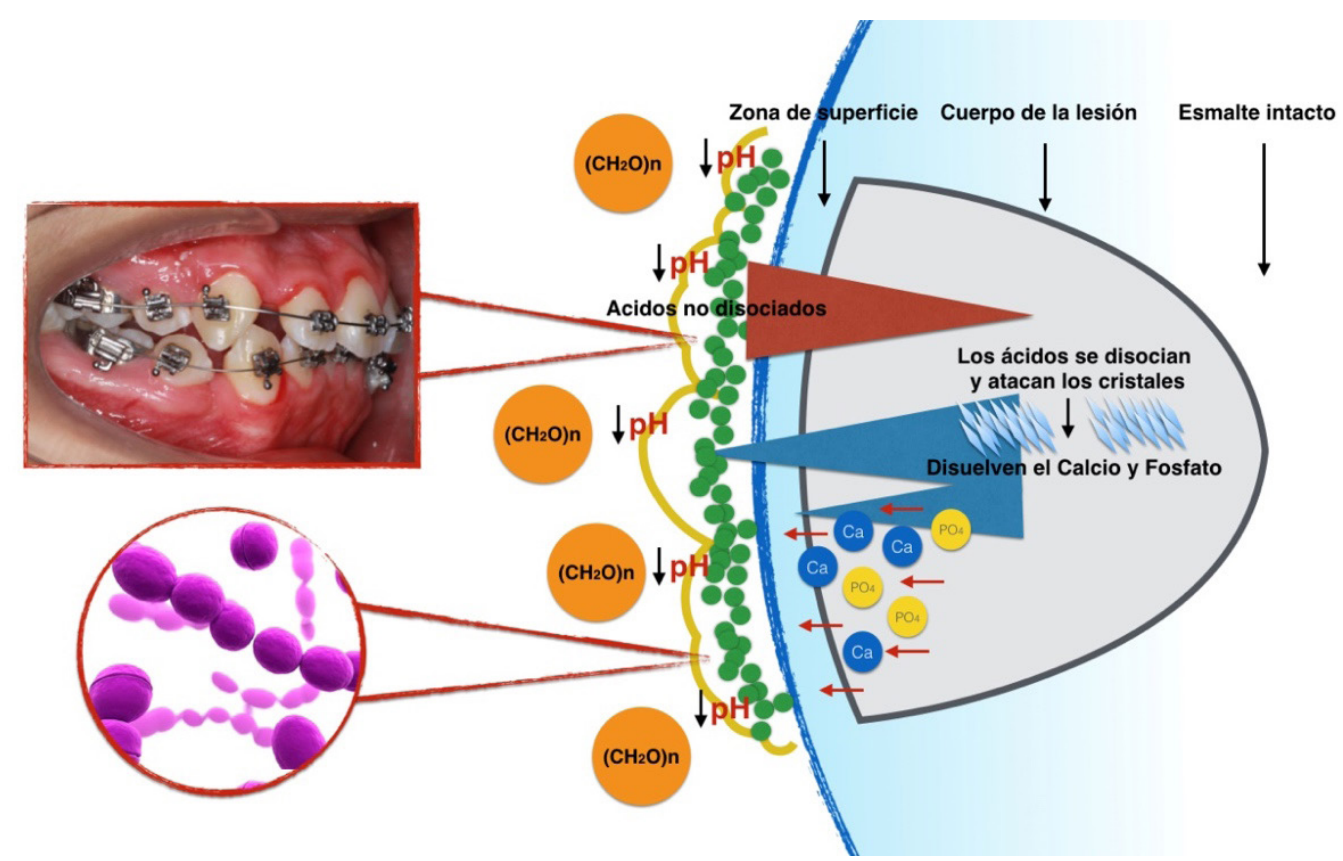

Figura 1. Esquema de la formación de las lesiones de mancha Blanca. Adaptado de: Zero DT,1999 (4). 
carbohidratos fermentables, disminuyendo el $\mathrm{pH}$ hasta alcanzar niveles críticos cercanos a 5,5 (4), los ácidos son capaces de difundirse dentro de la subsuperficie del esmalte a través de la película adquirida, disociándose y comenzando la desmineralización (figura 1).

La disolución del contenido mineral de la estructura del esmalte crea una alteración en el índice de refracción de la luz, lo que clínicamente se presenta como una opacidad blanco lechosa sobre la superficie del esmalte (5). Las LMB pueden remineralizarse y regresar a la normalidad, permanecer estable o progresar hacia la cavitación dependiendo del medio ambiente oral (6). Estas se pueden observar luego de un mes de haber cementado los aparatos ortodónticos fijos (3). Después del tratamiento, las LMB disminuyen dentro de los dos primeros años, pero pueden continuar siendo un problema estético que afecta a los pacientes luego del tratamiento ortodóntico (figura 2) (7).

\section{Prevalencia de las lesiones de mancha blanca en los pacientes ortodónticos}

La prevalencia de estas lesiones puede variar de $4,9 \%$ (8) al $84 \%$ (9). Esta gran variedad se debe a los diferentes métodos diagnósticos para las LMB, diferencias en el tamaño de la muestra estudiada, locación geográfica del estudio, duración del estudio, edad de los participantes al inicio del tratamiento y materiales empleado en el estudio. Tufekci et al., (10), evaluaron la prevalencia de lesiones de mancha blanca en pacientes ortodónticos a través de un método de examen visual, sus resultados mostraron que a los 12 meses de tratamiento la prevalencia era mayor en los pacientes varones que en las mujeres. Ritcher et al., (11) evaluaron fotografías pre y post tratamiento de

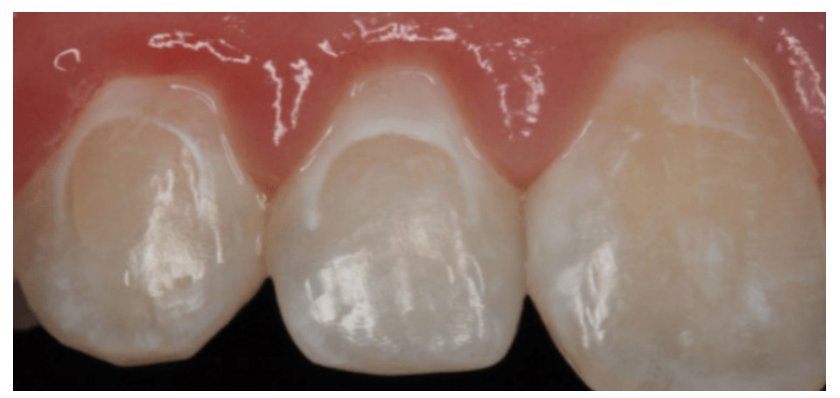

Figura 2. Lesiones de mancha blanca persistentes luego de la remoción de los brackets. ortodoncia encontrando una prevalencia de ocurrencia de lesiones de mancha blanca de $72,9 \%$. Empleando un método similar de evaluación de fotografías Julien et al., (12) encontraron una prevalencia del 32\%; siendo que los pacientes varones fueron los más afectados y la ocurrencia de las LMB se asociaba a tiempos prolongados de tratamiento y niveles bajos de higiene oral durante el tratamiento, datos similares han sido reportados por Masarwa et al., (13) y Benkaddoir et al., (14). Eltayeb et al., (15) evaluaron la prevalencia, el patrón de distribución y los factores contribuyentes para el desarrollo de las LMB, la prevalencia total fue del $61,4 \%$ y los dientes más afectados fueron los caninos con $48,1 \%$ de las lesiones y el tiempo prolongado de tratamiento fue la variable asociada a la ocurrencia de las LMB. Recientemente Admakin et al., (16) analizaron la prevalencia de las LMB a través la valoración del ICDAS, sus resultados mostraron que para aquellos pacientes con 6 meses de tratamiento ortodóntico, la prevalencia alcanzaba el 21\% y esta se incrementó al $44 \%$ a los 12 meses de tratamiento.

\section{Factores de riesgo asociados a las lesiones de mancha blanca}

En cuanto a los factores de riesgo para la formación de LMB, los estudios no han demostrado una predisposición clara con respecto al género de los pacientes, algunos muestran que los varones desarrollan más LMB que las mujeres y que incluso la severidad de las LMB es mayor $(10,12)$ y otros estudios presentan hallazgos discordantes, la aparente influencia del género puede ser en realidad debida al cumplimiento del paciente con las recomendaciones de higiene y prevención (17). La edad sí parece estar relacionada con la prevalencia de las LMB, Al Maaitah et al., (18), y Chapman et al.,(17) encontraron que los pacientes más jóvenes desarrollan más LMB que los pacientes adultos, e incluso la severidad de las mismas es mayor en los pacientes menores de 13 años (19).

Respecto a la localización de las LMB el arco maxilar parece tener un mayor riesgo que la mandíbula $(8,12)$, y los incisivos laterales superiores han sido reportados como los dientes con mayor frecuencia de aparición de las LMB (figura 3) (17). En el arco inferior el diente más afectado es el canino (20). Gorelick et al., (8) señalan que la reducida 


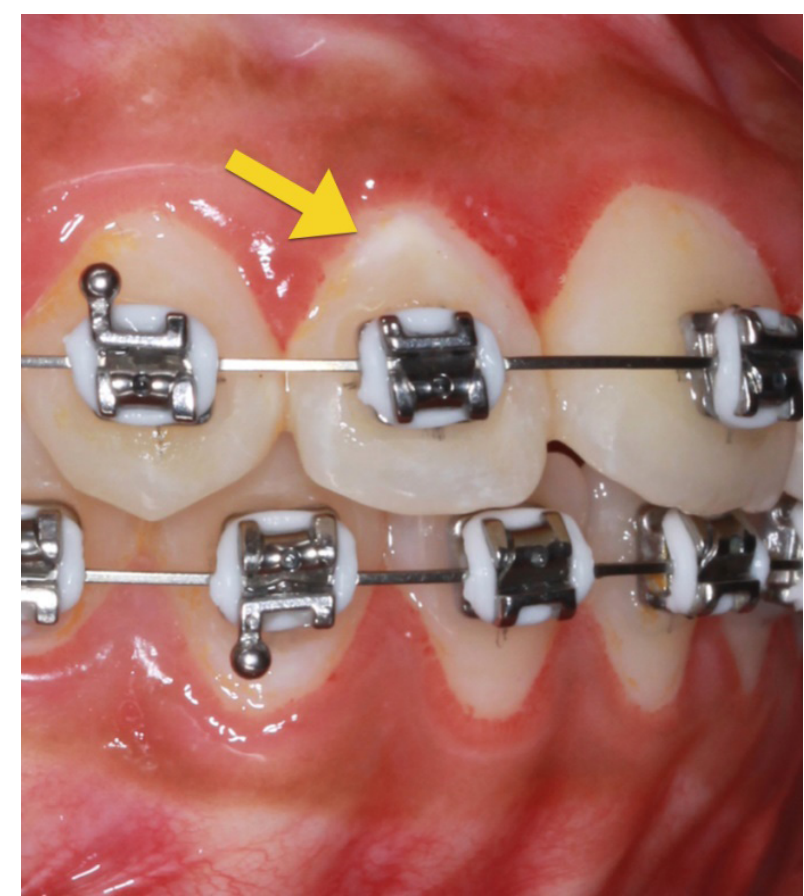

Figura 3. Detalle de lesión de mancha blanca en región cervical de pieza 1.2.

distancia entre el bracket y la encía libre que se observa en los dientes pequeños como los incisivos laterales explicaría la mayor frecuencia de ocurrencia de LMB, debido a que facilitaría el acúmulo de placa bacteriana y dificultaría el flujo normal de la saliva impidiendo el proceso de remineralización. Esto último, Propiciaría que las zonas adyacentes a los márgenes gingivales y alrededor de los brackets sean las localizaciones más frecuentes de las LMB (21).

La duración del tratamiento ortodóntico parece estar estrechamente relacionado con la aparición y severidad de las LMB. Julien et al., (12), encontraron una frecuencia muy alta de lesiones en aquellos pacientes con tratamientos con duración mayor a 36 meses comparados con aquellos de menor duración; además los pacientes con una pobre higiene oral antes y durante el tratamiento tienen un gran riesgo de desarrollar LMB, resultados similares han sido reportados por Eltayeb et al., (15). Boersma et al., (22), encontraron una relación directa entre los niveles de sangrado gingival y la presencia y severidad de las LMB. Pinto et al., (23), señalan que mientras más prolongado el tratamiento de ortodoncia, es mayor la prevalencia, extensión y severidad de lesiones de caries activas en pacientes adolescentes y adultos jóvenes. La experiencia previa de caries también está relacionada con la aparición de LMB durante el tratamiento de ortodoncia, Al Maaitah et al., (18) y Al Mulla et al., (24), encontraron que la presencia de caries, dientes restaurados o perdidos incrementa el riesgo de desarrollo y severidad de las LMB durante el tratamiento.

Los estudios coinciden que existe un alto riesgo de formación de las LMB cuando los tratamientos son prolongados y cuando existe un bajo índice de higiene bucal previo o durante el curso del tratamiento (12) (15)(17), otros factores no han podido demostrar una relación directa con la formación de las LMB.

\section{Efecto del tratamiento ortodóntico sobre el medio bucal}

Los aparatos ortodónticos incrementan la acumulación de placa sobre la superficie de los dientes, que en condiciones normales tendrían un bajo riesgo de experimentar la formación de lesiones de caries $(3,25)$. La presencia de los brackets dificulta el cepillado y el propio mecanismo de limpieza de la saliva. Se ha demostrado que el $\mathrm{pH}$ salival en los pacientes ortodónticos es menor que en aquellos que no llevan brackets (figura 4) (26,27). Además, el tratamiento ortodóntico altera la flora bacteriana de la placa al promover un descenso en el $\mathrm{pH}$, propiciando un incremento de las bacterias acidogénicas como el Streptococcus mutans y el Lactobacillus acidophilus (28). Cuando el pH se encuentra por debajo del nivel crítico, el inicio del proceso de desmineralización

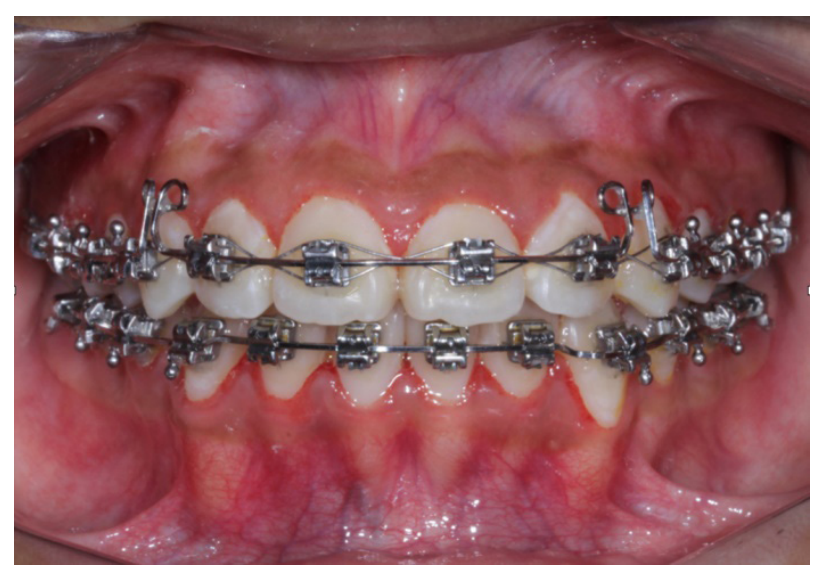

Figura 4. Detalle de paciente con tratamiento ortodóntico y formación inicial de lesiones de mancha blanca asociada a mala higiene oral. 
se ve facilitado (29). Por otro lado, Lara-Carrillo et al., (30), demostraron que luego de la colocación de los brackets el flujo salival aumenta, incrementando los niveles de $\mathrm{pH}$ y mejora la capacidad buffer de la misma. Estos cambios en la saliva podrían explicar por qué algunos pacientes no presentan desmineralizaciones a pesar del acúmulo de la placa bacteriana (31).

\section{Evaluación de las lesiones de mancha blanca en los pacientes ortodónticos}

\section{Inspección visual}

El método más común para la detección de las LMB es la inspección visual, se puede emplear lupas de magnificación de 2,5x para mejorar la detección de las lesiones (32). Gorelick et al., (8) proponen un índice para detección de las LMB, incluyendo la presencia, ausencia y severidad, pero no indica cual área del diente es la que está afectada por la lesión. Banks et al., (33) proponen una escala numérica que incluye la evaluación del área afectada. Sin embargo, los métodos de inspección visual requieren que los observadores sean correctamente calibrados para la identificación de las lesiones, para que estas metodologías sean reproducibles y confiables.

\section{ICDAS II}

El sistema de detección y evaluación internacional de caries o ICDAS por sus siglas en inglés fue desarrollado en 2003 y luego modificado a ICDAS II en 2007, se trata de un índice visual de detección de caries; su ventaja es que permite una mejor categorización de las lesiones de desmineralización inicial del esmalte $(34,35)$. Esto es particularmente importante para las LMB debido que, al tratarse de lesiones iniciales de caries, requieren criterios más rigurosos para su identificación y diferenciación. Los códigos y criterios de clasificación pueden ser observados en la tabla 1.

\section{Fluorescencia Láser}

La fluorescencia es la emisión de luz debido al movimiento de moléculas en respuesta a la absorción de luz de alta energía (32). Los dientes presentan fluorescencia natural y esta se debe a las proteínas presentes en el esmalte y la dentina (36). La fluorescencia laser (FL) es un método cuantitativo para la detección de caries basado en la emisión de luz a partir de un diodo laser $(\lambda=655 \mathrm{~nm})$ y el consecuente registro de la fluorescencia emitida por el diente (37). Los metabolitos bacterianos producidos en las lesiones de caries emiten fluorescencia que la FL puede medir. Las LMB pueden ser detectadas por este método, siendo más sensible que la inspección visual (38).

\section{Imágenes fotográficas}

El empleo de las fotografías es un método bastante empleado para la detección de las LMB $(11,12)$. Se basa en el registro fotográfico antes de iniciar el tratamiento de ortodoncia y compararlo con las fotografías tomadas luego de un periodo de tiempo que suele ser de 6 a 12 meses en búsqueda de zonas opacas en el esmalte adyacentes a los brackets que corresponderían a la formación de las LMB. Sin embargo, para que este método sea efectivo se deben controlar factores técnicos como una correcta iluminación, evitar que se produzcan reflejos que podrían confundirse con las LMB. Además, los

Tabla 1. El sistema de detección y evaluación internacional de caries - ICDAS II (34).

\begin{tabular}{ll}
\hline Código & Criterio \\
\hline 0 & Superficie del diente sana: sin evidencia de caries después de secar 5 segundos con aire. \\
1 & Primer cambio visual en el esmalte. Descoloración opaca es visible luego de secado prolongado con aire. \\
2 & Cambios visuales diferenciados en el esmalte visible con humedad. La lesión debe ser visible al secarse. \\
3 & $\begin{array}{l}\text { Falla localizada del esmalte (sin signos visuales clínicos de afectación dentinal) vista al estar mojado y des- } \\
\text { pués de un secado prolongado }\end{array}$ \\
5 & Sombra oscura subyacente de dentina \\
6 & Cavidad diferenciada con dentina visible \\
\hline
\end{tabular}


ángulos con que las fotos son tomadas debe ser similar entre cada fotografía para poder ser comparables entre ellas (39).

\section{Luz cuantitativa inducida por fluorescencia}

La luz cuantitativa inducida por fluorescencia o QLF (Quantitative light-induced fluorescence) por sus siglas en inglés, ha sido probada tanto en estudios in vitro $(0,41)$ como in vivo $(42)$ para la detección de las LMB en pacientes ortodónticos. Esta técnica no solo detecta las lesiones tempranas, sino que puede detectar cambios en la perdida mineral a través de periodos de tiempo, debido a que el software con el que trabaja guarda la información de las imágenes registradas. El dispositivo emplea una lámpara de arco con una guía de luz, la luz pasa a través de un filtro azul con una intensidad de $370 \mathrm{~nm}$, un filtro de paso es mantenido frente a la cámara de detección para excluir la luz azul dispersa y la combinación es optimizada para que existan reflejos (43).

\section{Prevención y tratamiento de las lesiones de mancha blanca}

El manejo de las LMB involucra tanto la prevención como el tratamiento. En la literatura diversos métodos preventivos han sido descritos como: el uso de flúor, clorhexidina, sellantes y adhesivos con inclusión de diversos materiales con propiedades antibacterianas. Los pacientes bajo tratamiento ortodóntico deben recibir instrucciones de higiene oral, indicaciones de reducción del consumo de dietas ricas en carbohidratos, bebidas ácidas o carbonatadas, con el objetivo de mantener un bajo riesgo de caries (44).

\section{Flúor}

La aplicación de flúor durante el tratamiento ortodóntico puede ser tópica (enjuague, barniz, gel, pasta, etc.,) o incorporada a algún material ortodóntico (cemento, adhesivo, etc.,). Cuando el flúor se incorpora al esmalte, se forman cristales de fluorapatita que presentan una mayor resistencia al ataque ácido que los cristales de hidroxiapatita (29). Ogaard et al., (45), demostraron que la aplicación de enjuague o barniz de flúor durante el tratamiento ortodóntico reduce la frecuencia y severidad de las LMB. Materiales con la capacidad de liberar flúor también han sido desarrollados, particularmente las resinas modificadas con ionómero de vidrio (RMIV) pueden prevenir la desmineralización alrededor de los brackets, pero su desventaja radica en que los niveles de liberación de flúor caen rápidamente a niveles subterapéuticos, requiriendo el uso combinado de enjuagues para mantener niveles apropiados que permitan la liberación sostenida de flúor (46), además las RMIV presentan menores niveles de fuerzas de adhesión al esmalte dentario que las resinas convencionales, lo que puede comprometer la adherencia de los brackets $(47,48)$.

\section{Fosfato de calcio amorfo}

El fosfato de calcio amorfo (FCA) o el fluoruro fosfato de calcio amorfo (FFCA) poseen efecto remineralizador sobre la superficie del esmalte (49). Uysal et al., (50), incorporaron FCA a una resina para ortodoncia y observaron que existió una reducción en la perdida mineral alrededor de los brackets al compararlas con una resina convencional. Por otro lado, las resinas a las que se les ha incorporado FCA presentan menor adhesión al esmalte (51).

\section{Sellantes}

Los sellantes aplicados alrededor de los brackets actúan como una barrera física contra el ataque ácido. Estos sellantes pueden ser combinados con flúor o agentes antibacterianos mejorando sus propiedades contra la desmineralización; sin embargo, al poseer baja carga son poco resistentes a la abrasión del cepillado y pueden requerir ser reaplicados frecuentemente (52).

\section{Agentes antibacterianos}

Una reciente estrategia para el control de las LMB es la incorporación de agentes antibacterianos a los cementos para la adhesión de brackets, como el óxido de zinc (53), nanopartículas de plata (54), cloruro de benzalconio $(55,56)$, cloruro de cetilpiridinio (57), cloruro de trimetilamonio (58), nanopartículas de óxido de titanio (59), nanopartículas de óxido de cobre (60), asociación de nanopartículas de plata e hidroxiapatita (61), nanorellenos de sílica y nanopartículas de plata (62), nanopartículas de cúrcuma (63) y extracto de Galla Chinensis (64). Lo interesante de estas propuestas es que parecen no 
tener efectos citotóxicos ni afectar las propiedades mecánicas de los cementos, lo que facilitaría su empleo clínico eliminando la necesidad de colaboración de los pacientes con la ventaja de controlar la aparición de las LMB.

\section{CONCLUSIONES}

La desmineralización del esmalte alrededor de los brackets continúa siendo uno de los efectos adversos más prevalentes durante el tratamiento de ortodoncia. Los factores de riesgos más importantes para la aparición de las lesiones de mancha blanca son la mala higiene bucal y tiempos prolongados de tratamiento. Finalmente, las propuestas de manejo para las LMB deben empezar por enfoques preventivos y menos invasivos con el objetivo de no alterar la estructura del esmalte.

Conflicto de intereses: Todos los autores declaran que no tienen conflicto de intereses

Financiamiento: Ninguno.

Contribuciones de los autores: Todos los autores contribuyeron a este manuscrito.

\section{Correspondencia:}

Lidia Yileng Tay Chu Jon

Facultad de Estomatología

Dirección de Postgrado y Especialización

Av. Salaverry N²475 San Isidro, Lima.

Correo electrónico: lidia.tay.c@upch.pe

\section{REFERENCIAS BIBLIOGRÁFICAS}

1. Zachrisson BU, Zachrisson S: Caries incidence and oral hygiene during orthodontic treatment. Scan J Dent Res. 1971;79:394-401.

2. O'Reilly MM, Featherstone JDB. Demineralization and remineralization around orthodontic appliances: an in vivo study. Am J Orthod Dentofacial Orthop. 1987; 92(1):33-40.

3. Ogaard B, Rolla G, Arends J. Orthodontic appliances and enamel demineralization. Part 1. Lesion development. Am J Orthod Dentofacial Orthop. 1988;94(1):68-73.

4. Zero DT. Dental caries process. Dental Clin North Am. 1999;43(4):635-664.

5. Summitt JB, Robbins JW, Schwartz RS. Fundamentals of Operative Dentistry: A Contemporary Approach. Hanover Park, IL: Quintessence Publishing, 2006.p. $2-4$.
6. Shungin D, Olsson Al, Pesson M. Orthodontic treatment-related white spot lesions: a 14-year prospective quantitative follow-up, including bonding material assessment. Am J Orthod Dentofacial Orthop. 2010;138(2):136e1-138.

7. Mattousch TJ, Van-der-Veen MH, Zentner A. Caries lesions after orthodontic treatment followed by quantitative light-induced fluorescence: a 2-year follow-up. Eur J Orthod. 2007;29(3):294-298.

8. Gorelick L, Geiger AM, Gwinnett AJ. Incidence of white spot formation after bonding and banding. Am J Orthod. 1982;81(2):93-98.

9. Mizrahi E: Enamel demineralization following orthodontic treatment. Am J Orthod Dentofacial Orthop. 1982;82(1):62-67.

10. Tufekci E, Dixon JS, Gunsolley JC, Lindauer SJ. Prevalence of white spot lesions during orthodontic treatment with fixed appliances. Angle Orthod. 2011;81(2):206-210.

11. Richter EA, Arruda OA, Peters CM, Sohn W. Incidence of caries lesions among patients treated with comprehensive orthodontics. Am J Orthod Dentofacial Orthop. 2011;139(5):657-664.

12. Julien KC, Buschabg PH, Campbell PM. Prevalence of white spot lesion formation during orthodontic treatment. Angle Orthod. 2013;83(4):641-647.

13. Masarwa AN, Al-Nsour FH, Al-Zoubi HZ, Al-Awabdeh FH, Al-Khraisat SA. Prevalence of new carious lesions among patients undergoing orthodontic treatment with fixed appliances. Pak Oral Dent J. 2013;33(3):539-543.

14. Benkaddour A, Bahije L, Bahoum A, Zaoui F. Orthodontics and enamel demineralization: clinical study of risk factors. Int Orthod. 2014;12(4):458-466.

15. Eltayeb KM, Ibrahim EY, El Karim AI, Sanhouri MN. Distribution of white spot lesions among orthodontic patients attending teaching institutes in Khartoum. BMC Oral Health.2017;17(1):88.

16. Admakin O, Khakimova D, Slop I. Prevalence white spot lesions in patients with fixed orthodontic appliances. IAJPS. 2018; 5(5):4640-4643.

17. Chapman JA, Roberts WE, Eckert GJ, Kula KS, Gonzales-Cabezas C. Risk factors for incidence and severity of white spot lesions during treatment with fixed appliances. Am J Orthod Dentofacial Orthop. 2010;138(2):188-194.

18. Al maaitah EF, Adeyemi AA, Higham SM, Pender N, Harrison JE. Factors affecting demineralization during orthodontic treatment: a post-hoc analysis of RCT recruits. Am J Orthod Dentofacial Orthop. 2011;139(2):181-191.

19. Geiger AM, Gorelick L, Gwinnett AJ, Griswold PG. The effect of a fluoride program on white spot formation during orthodontic treatment. Am J Orthod Dentofacial Orthop. 1988; 93(1):29-37.

20. Travess H, Roberts-Harry D, Sandy J. Orthodontics. 
Part 6: Risks in orthodontic treatment. Br Dent J. 2004; 196(2):71-77.

21. Mitchell L. Decalcification during orthodontic treatment with fixed appliances - an overview. $\mathrm{Br} \mathrm{J}$ Orthod. 1992;19(3):199-205.

22. Boersma JG, Van der Vee MH, Lagerweij MD, Bokhout B, Prahl-Anderser B. Caries prevalence measured with QLF after treatment with fixed orthodontic appliances: influencing factor. Caries Res. 2005;39(1):41-47.

23. Pinto SA, Alves SL, Maltz M, Susin C. Does the duration of fixed orthodontic treatment affect caries activity among adolescents and Young adults?. Caries Res. 2018;52:463-467.

24. Al Mulla AH, Kharsa SA, Kjellberg H, Birkhed D. Caries risk profiles in orthodontic patients at follow-up using cariogram. Angle Orthod. 2009; 79(2):323-330.

25. Lovrov S, Hertrich K, Hirschfelder U. Enamel Demineralization during fixed orthodontic treatment - incidence and correlation to various oral hygiene parameters. J Orofac Orthop. 2007; 68(5):353-363.

26. Chatterjee R, Kleinberg I. Effect of orthodontic band placement on the chemical composition of human incisor tooth plaque. Arch Oral Biol. 1979; 24(2):97100.

27. Lundstrom F, Krasse B. Caries incidence in orthodontic patients with high levels of streptococcus mutans. Eur J Orthod. 1987; 9(2):117-121.

28. Attin R, Thon C, Schlagenhauf U, et al. Recolonization of mutans streptococci on teeth with orthodontic appliances after antimicrobial therapy. Eur J Orthod. 2005; 27(5):489-493.

29. Bishara SE, Ostby AW. White spot lesions: formation, prevention and treatment. Semin Orthod. 2008; 14(3):174-182.

30. Lara-Carrillo E, Montiel-Bastida NM, SanchezPerez L, Alanis-Tavira J. Effect of orthodontic treatment on saliva, plaque and the levels of streptococcus mutans and lactobacillus. Med Oral Patol Oral Cir Bucal. 2010; 15(6):e924-929.

31. Ulukapi H, Koray F, Efes B. Monitoring the caries risk of orthodontic patients. Quintessence Int. 1997; 28(1):27-29.

32. Guerrieri A, Gaucher C, Bonte E, Lasfargues JJ. Minimal intervention dentistry: part 4. Detection and diagnosis of initial caries lesions. Br Dent J. 2012; 213(11):551-557.

33. Banks PA, Chadwick SM, Asher-McDade C, Wright JL. Fluoride-releasing elastomerics - a prospective controlled clinical trial. Eur J Orthod. 2000; 22(4)401407.

34. Ismail Al, Sohn W, Tellez M, Amaya A, Sen A, Hasson $\mathrm{H}$, et al. The International Caries Detection and Assessment System (ICDAS): an integrated system for measuring dental caries. Community Dent Oral Epidemiol. 2007; 35(3):170-178.
35. Shoaib L, Deery C, Ricketts DN, Nugent ZJ. Validity and reproducibility of ICDAS II in primary Teeth. Caries Res. 2009; 43(6):442-448.

36. Lussi A, Hibst R, Paulus R. DIAGNOdent: an optical method for caries detection. J Dent res. 2004; 83 Spec N C:C80-3.

37. Alencar CJ, Braga MM, de Oliveira E, Nicolau J, Mendes FM. Dye-enhanced laser fluorescence detection of caries lesions around brackets. Lasers med Sci. 2009; 24(6):865-870.

38. Aljehani A, Yousif MA, Angmar-Mansson B, Shi XQ. Longitudinal quantification of incipient carious lesions in postorthodontic patients using a fluorescence method. Eur J Oral Sci. 2006;114(5):430-434.

39. Benson PE, Shah AA, Willmot DR. Measurement of white lesions surrounding orthodontic brackets: captured slides vs digital camera images. Angle Orthod. 2005;75(2):226-230.

40. Benson PE, Pender N, Higham SM: Quantifying enamel demineralization from teeth with orthodontic bracketsa comparison of two methods. Part 1: repeatability and agreement. Eur J Orthod. 2003;25(2):159-165.

41. Restrepo M, Bussaneli GD, Jeremias F,et al. Control of white spot lesions with use of fluoride varnish or chlorhexidine gel during orthodontic treatment a randomized clinical trial. J Clin Pediatr Dent. 2016;40(4):274-280.

42. Van-der-Veen MH, Mattousch T, Boersma JG. Longitudinal development of caries lesions after orthodontic treatment evaluated by quantitative lightinduced fluorescence. Am J Orthod Dentofac Orthop. 2007;131(2):223-228.

43. Benson P. Evaluation of White spot lesions on teeth with orthodontic brackets. Semin Orthod. 2008; 14(3):200-208.

44. Noble J, Cassolato S, Karaikos N, Wiltshire WA. Point of care. Preventive and interceptive measures for improving and maintaining good oral hygiene and cariogenic control in orthodontic patients. J Can Dent Assoc. 2009;75(6):441-443.

45. Ogaard B, Larsson E, Henriksson T, Birkhed D, Bishara SE. Effects of combined application of antimicrobial and fluoride varnishes in orthodontic patients. Am J Orthod Dentofacial Orthop. 2001;120(1):28-35.

46. Benson PE, Parkin N, Millett DT, Dyer FE, Vine S, Shah A. Fluorides for the prevention of White spot on teeth during fixed brace treatment. Cochrane Database Syst Rev. 2004(3):CD003809

47. Krbmacher HM, Huck L, Kahl-Nieke B. Fluoridereleasing adhesive and antimicrobial sefl-etching primer effects on shear bond strength of orthodontic brackets. Angle Orthod. 2006; 76(5):845-850.

48. Graf I, Jacobi BE. Bond strength of various fluoridereleasing orthodontic bonding systems. Experimental study. J Orofac Orthop. 2000;61(3):191-198. 
49. Bailey DL, Adams GG, Tsao CE, Hyslop A, Escobar K, Manton DJ, et al. Regression of post-orthodontic lesions by a remineralizing cream. J Dent Res. 2009;88 (12):1148-53.

50. Uysal T, Amasyali M, Ozcan S, Koyuturk AE, Akyol M, Sagdic D. In vivo effects of amorphous calcium phosphate-containing orthodontic composite on enamel demineralization around orthodontic brackets. Aust Dent J. 2010;55(3):285-291.

51. Foster JA, Berzins DW, Bradley TG. Bond strength of an amorphouscalciumphosphate.containingorthodontic adhesive. Angle Orthod. 2008;78(2):339-344.

52. Knosel M, Forslund L, Jung K, Ziebolz D. Efficacy of different strategies in protecting enamel against demineralization during fixed orthodontic treatment. J Orofac Orthop. 2012;73(3):194-203.

53. Spencer GC, Campbell MP, Buschang HP, Cai J, Honeyman LA. Antimicrobial effects of zinc oxide in a orthodontic bonding agent. Angle Orthod. 2009; 79(2):317-322.

54. Wang X, Wang B, Wang Y. Antibacterial orthodontic cement to combat biofilm and White spot lesions. Am J Orthod Dentofacial Orthop. 2015;148(6):974-981.

55. Othman HF, Wu CD, Evans CA, Drummond JL, Matasa CG. Evaluation of antimicrobial properties of orthodontic composite resins combined with benzalkonium chloride. Am J Orthod Dentofacial Orthop. 2002;122(3):288-294.

56. Saito K, Hayakawa T, Kawabata R, Meguro D, Kasai $\mathrm{K}$. In vitro antibacterial and cytotoxicity assessments of an orthodontic bonding agent containing benzalkonium chloride. Angle Orthod. 2009;79(2):331337.

57. Al-Musallam TA, Evans CA, Drummond JL, Matasa $\mathrm{C}, \mathrm{Wu} \mathrm{CD}$. Antimicrobial properties of an orthodontic adhesive combined with cetylpyridinium chloride. Am J Orthod Dentofacial Orthop. 2006; 129(2):245-251.
58. Nascimento PLMM, Meereis CTW, Maske TT, Ogliari FA, Cenci MS, Pfeifer CS, Faria-E-Silva A. Addition of ammonium-based methacrylates to an experimental dental adhesive for bonding metal brackets: Carious lesion development and bond strength after cariogenic challenge. Am J Orthod Dentofacial Orthop. 2017;151(5):949-956.

59. Poosti M, Ramazanzadeh B, Zebarjad M, Javadzadeh P, Naderinasab M, Shakeri MT. Shear bond strength and antibacterial effects of orthodontic composite containing TiO 2 nanoparticles. Eur J Orthod. 2013;35(5):676679.

60. Toodehzaeim MH, Zandi H, Meshkani H, Firouzabadi AH. The Effect of CuO Nanoparticles on Antimicrobial Effects and Shear Bond Strength of Orthodontic Adhesives. J Dent (Shiraz). 2018;19(1):1-5.

61. Sodagar A, Akhavan A, Hashemi E, et al. Evaluation of the antibacterial activity of a conventional orthodontic composite containing silver/hydroxyapatite nanoparticles. Prog Orthod. 2016;17(1):40.

62. Ahn SJ, Lee SJ, Kook JK, Lim BS. Experimental antimicrobial orthodontic adhesives using nanofillers and silver nanoparticles. Dent Mater. 2009(2):206-213.

63. Sodagar A, Bahador A, Pourhajibagher M, Ahmadi B, Baghaeian P. Effect of addition of curcumin nanoparticles on antimicrobial property and shear bond strength of orthodontic composite to bovine enamel. J Dent (Tehran). 2016;13(5):373-382.

64. Wang LF, Luo F, Xue CR, Deng M, Chen C, Wu H. Antibacterial effect and shear bond strength of an orthodontic adhesive cement containing Galla chinensis extract. Biomed Rep. 2016;4(4):507-511.

Recibido : 28-02-2020

Aceptado : 10-12-2020 